\title{
On Linear Hamiltonian Systems
}

\author{
Tatiana Titova ${ }^{1, *}$ \\ ${ }^{1}$ Moscow State University of Civil Engineering, Yaroslavskoe shosse, 26, Moscow, 129337, Russia
}

\begin{abstract}
In this paper we consider the normalization of quadratic Hamiltonian. We get the new method to find the generating function of the canonical transformation. We obtain the solution of the system of matrix equations to find this transformation. The corresponding Hamiltonian matrix has multiply eigenvalues.
\end{abstract}

\section{Introduction}

The Hamiltonian systems have an important role in fluid mechanics. The systems are of potential interest for statistical mechanics. Let us consider the quadratic Hamiltonian $H=$ $=1 / 2 \boldsymbol{w}^{T} \Omega w$. By $\boldsymbol{w}$ denote vector with dimension $2 n$, by $\Omega$ denote the symmetric $2 n$-by- $2 n$ matrix. We consider the Hamiltonian system of differential equations

$$
\frac{d \mathbf{w}}{d t}=J \Omega \mathbf{w}, J=\left(\begin{array}{cc}
O_{n} & E_{n} \\
-E_{n} & O_{n}
\end{array}\right),
$$

where $E_{n}$ is identity matrix. With canonical transformation we find the normal form of Hamiltonian matrix $J \Omega$ :

$$
I=\left(\begin{array}{cc}
U & O_{n} \\
O_{n} & -U^{T}
\end{array}\right),
$$

where submatrix $U$ has Jordan normal form.

In the paper [1] the author considers the normalization of Hamiltonian matrix in the case of nonzero eigenvalues. In the paper [2] the author proves the possibility of normalization of non-singular Hamiltonian matrix. In the papers [3 - 5] the authors obtain other methods of normalization the matrix. We find the canonical transformation with generation function. Hamiltonian matrix has multiply nonzero and zero eigenvalues.

\section{System of matrix equations}

Let us consider the quadratic Hamiltonian

$$
H=1 / 2 \boldsymbol{w}^{T} \Omega \boldsymbol{w}=1 / 2 \boldsymbol{x}^{T} A \boldsymbol{x}+\boldsymbol{x}^{T} B \boldsymbol{y}+1 / 2 \boldsymbol{y}^{T} C \boldsymbol{y},
$$

\footnotetext{
*Corresponding author: tat titova@mail.ru
} 
where both $\boldsymbol{x}$ and $\boldsymbol{y}$ are vectors with dimension $n, \boldsymbol{w}=\left(\begin{array}{l}\boldsymbol{x} \\ \boldsymbol{y}\end{array}\right)$. By $A, B, C$ denote $n$-by- $n$ real matrices. The matrices $A$ and $C$ are symmetric. The matrix

$$
V=J \Omega=\left(\begin{array}{cc}
B^{T} & C \\
-A & -B
\end{array}\right)
$$

is corresponding Hamiltonian matrix. Let $\boldsymbol{q}, \boldsymbol{p}$ be vectors of new canonical coordinates with the same dimension $n$. Let us consider the generation function of the canonical transformation:

$$
S(\boldsymbol{x}, \boldsymbol{p})=1 / 2 \boldsymbol{p}^{T} K \boldsymbol{p}+\boldsymbol{p}^{T} L \boldsymbol{x}+1 / 2 \boldsymbol{x}^{T} M \boldsymbol{x},
$$

where the $n$-by- $n$ matrices $K, M$ are symmetric, $n$-by- $n$ matrix $L$ is non-singular. Since $\frac{\partial S}{\partial \boldsymbol{p}}=\boldsymbol{q}, \frac{\partial S}{\partial \boldsymbol{x}}=\boldsymbol{y}$, we obtain the system of equations:

$$
K \boldsymbol{p}+L \boldsymbol{x}=\boldsymbol{q}, L^{T} \boldsymbol{p}+M \boldsymbol{x}=\boldsymbol{y} .
$$

Therefore, the following equation takes place:

$$
\left(\begin{array}{l}
\boldsymbol{x} \\
\boldsymbol{y}
\end{array}\right)=\left(\begin{array}{cc}
L^{-1} & L^{-1} K \\
M L^{-1} & L^{T}-M L^{-1} K
\end{array}\right)\left(\begin{array}{l}
\boldsymbol{q} \\
\boldsymbol{p}
\end{array}\right)=T\left(\begin{array}{l}
\boldsymbol{q} \\
\boldsymbol{p}
\end{array}\right)
$$

We want to find an invertible matrix $T$ such that $T^{-1} V T=I$. From the equations (3), (5) it follows that

$$
\begin{gathered}
V T=\left(\begin{array}{cc}
B^{T} & C \\
-A & -B
\end{array}\right)\left(\begin{array}{cc}
L^{-1} & L^{-1} K \\
M L^{-1} & L^{T}-M L^{-1} K
\end{array}\right)= \\
=\left(\begin{array}{cc}
B^{T} L^{-1}+C M L^{-1} & -B^{T} L^{-1} K+C L^{T}-C M L^{-1} K \\
-A L^{-1}-B M L^{-1} & A L^{-1} K-B L^{T}+B M L^{-1} K
\end{array}\right)
\end{gathered}
$$

From the equations (1), (5) it follows that

$$
T I=\left(\begin{array}{cc}
L^{-1} & L^{-1} K \\
M L^{-1} & L^{T}-M L^{-1} K
\end{array}\right)\left(\begin{array}{cc}
U & O_{n} \\
O_{n} & -U^{T}
\end{array}\right)=\left(\begin{array}{cc}
L^{-1} U & L^{-1} K U^{T} \\
M L^{-1} U & -L^{T} U^{T}+M L^{-1} K U^{T}
\end{array}\right)
$$

Since the block matrices are equal we obtain the system of matrix equations:

$$
\begin{aligned}
& B^{T} L^{-1}+C M L^{-1}=L^{-1} U,-A L^{-1}-B M L^{-1}=M L^{-1} U, \\
& -B^{T} L^{-1} K+C L^{T}-C M L^{-1} K=L^{-1} K U^{T}, \\
& A L^{-1} K-B L^{T}+B M L^{-1} K=-L^{T} U^{T}+M L^{-1} K U^{T}
\end{aligned}
$$

It is clear that the forth equation follows from the first, second and third equations. The equations

$$
M B^{T}+M C M=-A-B M, M C L^{T}=L^{T} U^{T}-B L^{T}
$$


follow from first and second equations. Therefore, we get the equivalent system:

$$
\begin{aligned}
& M C M+M B^{T}+B M+A=O, \\
& L\left(B^{T}+C M\right) L^{-1}=U, \\
& K U^{T}+L\left(B^{T}+C M\right) L^{-1} K=L C L^{T}
\end{aligned}
$$

Finally, we get the system of matrix equations:

$$
\begin{aligned}
& M C M+M B^{T}+B M+A=O, \\
& L\left(B^{T}+C M\right) L^{-1}=U, \\
& K U^{T}+U K=L C L^{T}
\end{aligned}
$$

In this system the unknown matrices $M$ and $K$ are symmetric, matrix $L$ is non-singular. From the first equation we find the matrix $M$. From the second equation we find the matrices $L$ and $U$. From the third equation we find the matrix $K$. Finally we find the normal form (1) of the Hamiltonian matrix (3). We get the new corresponding Hamiltonian:

$$
H(\boldsymbol{q}, \boldsymbol{p})=\boldsymbol{p}^{T} U \boldsymbol{q}
$$

Theorem. Suppose the generating function (4) of the canonical transformation has nonsingular matrix $L$. The quadratic Hamiltonian (2) has the normal form (9) with the canonical transformation (5) if and only if the matrices $K, L$ and $M$ of the generating function are the solutions of the system (8).

\section{Solution of the system of matrix equations}

The first equation of the system (8) is called [5 - 7] matrix Riccati equation:

$$
M C M+M B^{T}+B M+A=O
$$

Let us consider methods of solving this equation in various special cases.

Case 1 . We substitute matrices $C=E, B=O$ into the equation (10). Then we get the equation:

$$
M^{2}+A=O
$$

We want to find the symmetric root of the symmetric matrix. The symmetric matrix $A$ is similar to the diagonal matrix $L_{A}$ with real eigenvalues:

$$
A=F^{T} L_{A} F ; L_{A}=\left(\begin{array}{ccc}
\lambda_{1} & \ldots & 0 \\
\vdots & \ddots & \vdots \\
0 & \ldots & \lambda_{n}
\end{array}\right) ;
$$

where the matrix $F$ is the orthogonal matrix, by $\lambda_{i}$ we denote the eigenvalues of the matrix $A$. Let $\Lambda_{A}$ be a diagonal matrix such that $\Lambda_{A}^{2}=-L_{A}$ :

$$
\Lambda_{A}=\left(\begin{array}{ccc} 
\pm \sqrt{-\lambda_{1}} & \ldots & 0 \\
\vdots & \ddots & \vdots \\
0 & \ldots & \pm \sqrt{-\lambda_{n}}
\end{array}\right)
$$


The diagonal entries of the matrix $\Lambda_{A}$ are real or pure imaginary numbers. Therefore we get the symmetric solution of the equation (11):

$$
M=F^{T} \Lambda_{A} F
$$

Case 2. Let $C$ be a symmetric positive-definite matrix, $B=O$. We substitute these matrices into the equation (10). Then we get the following equation:

$$
M C M+A=O
$$

Let $Q$ be such that $C=Q Q^{T}$. After the change $M_{1}=Q^{T} M Q, A_{1}=Q^{T} A Q$ we get the following equation:

$$
M_{1}^{2}+A_{1}=O ; A_{1}^{T}=A_{1}
$$

If the matrix $M_{1}$ is the symmetric solution of the equation (14) then the matrix

$$
M=\left(Q^{T}\right)^{-1} M_{1} Q^{-1}
$$

is the symmetric solution of the equation (13).

Case 3. Let us substitute matrices $C=E, B=B^{T}$ into the equation (10). Then we get the equation:

$$
M^{2}+M B+B M+A=O
$$

After the change $M=N-B, D=B^{2}-A$ we get the equation $N^{2}=D$. Since matrix $D$ is symmetric matrix we obtain the same equation as the equation (11). Let the matrix $F$ be the orthogonal matrix such that $D=F^{T} L_{D} F$, where the matrix $\mathrm{L}_{D}$ is the diagonal matrix. Let the matrix $\Lambda_{D}$ be the diagonal matrix such that $\Lambda_{D}^{2}=L_{D}$. Then the matrix

$$
M=F^{T} \Lambda_{D} F-B
$$

is the symmetric solution of the equation

Case 4. Let us consider the equation:

$$
M^{2}+M B^{T}+B M+A=O
$$

After the change $M=N-B, M^{T}=N^{T}-B^{T}, D=B B^{T}-A$ we get the equation:

$$
N N^{T}=D
$$

The symmetric matrix $D$ is similar to the diagonal matrix $L_{D}$. Therefore $D=F^{T} L_{D} F$, where the matrix $F$ is the orthogonal matrix. Let the matrix $\Lambda_{D}$ be the diagonal matrix such that $\Lambda_{D}^{2}=L_{D}$. Then we get the solution of the equation (19):

$$
N=F^{T} \Lambda_{D} G
$$

where the matrix $G$ is any orthogonal matrix. Since $M$ is the symmetric matrix, we obtain the equality: $N-B=N^{T}-B^{T}$. After the change (20) we get the equation:

$$
F^{T} \Lambda_{D} G-G^{T} \Lambda_{D} F=B-B^{T}
$$

After the change $X=G F^{T}, P=F\left(B-B^{T}\right) F^{T}$ finally we get the equation:

$$
\Lambda_{D} X-X^{T} \Lambda_{D}=P \text {, }
$$


where the matrix $P$ is the skew-symmetric matrix. From the equation (21) we want to find the orthogonal complex matrix $X$. Let us see the matrices $X=\left(x_{i j}\right), P=\left(p_{i j}\right), \Lambda_{D}=$ $=\operatorname{diag}\left\{\lambda_{1}, \ldots, \lambda_{n}\right\}$. From the equation $(21)$ we get the system of $1 / 2 n(n-1)$ linear equations:

$$
\lambda_{i} x_{i j}-\lambda_{j} x_{j i}=p_{i j}, 1 \leq i<j \leq n
$$

After solving the system (22) we get the solution of the equation (18):

$$
M=F^{T} \Lambda_{D} X F-B
$$

Case 5. Let the matrix $C$ of the equation (10) be the symmetric positive-definite matrix, therefore $C=Q Q^{T}$. After the change

$$
M_{1}=Q^{T} M Q, \mathrm{~B}_{1}=Q^{T} B\left(Q^{T}\right)^{-1}, A_{1}=Q^{T} A Q
$$

we obtain the equation similar to the equation (18). Let $M_{1}$ be the solution of the equation

$$
M_{1}^{2}+M_{1} B_{1}^{T}+B_{1} M_{1}+A_{1}=O
$$

Then the matrix

$$
M=\left(Q^{T}\right)^{-1} M_{1} Q^{-1}
$$

is the symmetric solution of the equation (10).

Let us consider the second equation of the system (8). In this equation the matrix $U$ is the Jordan normal form of the matrix $B^{T}+C M$. Hence this equation always has the solution.

Let us consider the third equation of the system (8). The unknown matrix $K$ is symmetric. Let us consider matrices

$$
\begin{gathered}
U=\left(\begin{array}{cccc}
v_{1} & 0 & \cdots & 0 \\
\varepsilon_{1} & v_{2} & \cdots & 0 \\
\vdots & \vdots & \ddots & \vdots \\
0 & \cdots & \varepsilon_{n-1} & v_{n}
\end{array}\right), \varepsilon_{i}=0,1, i=\overline{1, n-1} ; \\
L C L^{T}=\left(c_{i j}\right), K=\left(k_{i j}\right) .
\end{gathered}
$$

From the third equation of the system (8) we get the system of $1 / 2 n(n+1)$ linear equations:

$$
\begin{gathered}
\left(v_{i}+v_{j}\right) k_{i j}+\varepsilon_{i-1} k_{i-1, j}+\varepsilon_{j-1} k_{i, j-1}=c_{i j}, k_{i j}=k_{j i}, \varepsilon_{0}=k_{0 j}=k_{i 0}=0, \\
1 \leq i \leq j \leq n .
\end{gathered}
$$

The determinant of the system is $\prod_{1 \leq i \leq j \leq n}\left(v_{i}+v_{j}\right)$. If the determinant is nonzero, then the system has the unique solution. We consistently find the unknowns of the system.

Thus we find the solution of the system of matrix equations.

Example. Let

$$
A=\left(\begin{array}{cc}
1 & -8 \\
-8 & 4
\end{array}\right), B=\left(\begin{array}{cc}
1 & -2 \\
-2 & 3
\end{array}\right), C=E
$$


be the matrices of the Hamiltonian (2). Therefore

$$
D=B^{2}-A=\left(\begin{array}{ll}
4 & 0 \\
0 & 9
\end{array}\right), \Lambda_{D}=\left(\begin{array}{ll}
2 & 0 \\
0 & 3
\end{array}\right) \text {. }
$$

Thus the matrices

$$
M=\Lambda_{D}-B=\left(\begin{array}{ll}
1 & 2 \\
2 & 0
\end{array}\right), L=E, U=\Lambda_{D}, K=\left(\begin{array}{cc}
1 / 4 & 0 \\
0 & 1 / 6
\end{array}\right)
$$

are the solution of the system (8). So

$$
\begin{aligned}
& S(\boldsymbol{x}, \boldsymbol{p})=1 / 2 \boldsymbol{p}^{T} K \boldsymbol{p}+\boldsymbol{p}^{T} L \boldsymbol{x}+1 / 2 \boldsymbol{x}^{T} M \boldsymbol{x}=1 / 8 p_{1}^{2}+1 / 12 \mathrm{p}_{2}^{2}+ \\
& +p_{1} x_{1}+p_{2} x_{2}+1 / 2 x_{1}^{2}+2 x_{1} x_{2}
\end{aligned}
$$

is the generation function of the canonical transformation. Hence

$$
H(\boldsymbol{q}, \boldsymbol{p})=\boldsymbol{p}^{T} U \boldsymbol{q}=2 p_{1} q_{1}+3 p_{2} q_{2}
$$

is the new Hamiltonian.

\section{Conclusions}

We obtain the new method of normalization of the quadratic Hamiltonian. With this method we can investigate the stability of the solution of the Hamiltonian systems.

\section{References}

1. J. Roels, G. Louterman, Normalisation des systèmes linéaires canoniques et application au problème restreint des trois corps, Celestial Mechanics 3, 1 (1970): p. 129-135.

2. A.J. Laub, K. Meyer, Canonical Forms for Symplectic and Hamiltonian Matrices, Celestial Mechanics 9, 2 (1974): p. 213-223.

3. A.D. Bryuno, A.G. Petrov, O vychislenii gamil'tonovoy normal'noy formy, Doklady Akademii Nauk, 410, 4 (2006): p. 474 - 478.

4. T.N. Titova, Svoystva gamil'tonovykh matrits, Estestvennye i tekhnicheskie nauki 84, 6 (2015): p. 65-66.

5. J.E. Potter, Matrix quadratic solutions, SIAM Journal of Applied Mathematics 14, 3 (1966): p. 496-499.

6. K. Martensson, On the matrix Riccaty equation, Information Science 3, 1 (1971): p. $17-23$.

7. L.G. Kurakin, I.V. Ostrovskaya, Nonlinear Stability Analysis of a Regular Vortex Pentagon Outside of a Circle, Regular and Chaotic Dynamics 17, 5 (2012): p. 385-396. 